\title{
Helical motions in the jet of blazar $1156+295$
}

\author{
X.Y. Hong ${ }^{a}$ D.R. Jiang ${ }^{a}$ L.I. Gurvits ${ }^{b}$ M.A. Garrett ${ }^{b}$ \\ R.T. Schilizzi ${ }^{b}$ R.D. Nan ${ }^{\mathrm{c}}$ \\ a Shanghai Astronomical Observatory, 80 Nandan Road, Shanghai, 200030, China \\ b Joint Institute for VLBI in Europe, Postbus 2, 7990 AA Dwingeloo, \\ The Netherlands \\ ${ }^{\mathrm{c}}$ Beijing Astronomical Observatory, Datun Rd. 20A, Chaoyang District, \\ Beijing 100012, China
}

\begin{abstract}
The blazar $1156+295$ was observed by VLBA and EVN + MERLIN at $5 \mathrm{GHz}$ in June 1996 and February 1997 respectively. The results show that the jet of the source has structural oscillations on the milliarcsecond scale and turns through a large angle to the direction of the arcsecond-scale extension. A helical jet model can explain most of the observed properties of the radio structure in $1156+295$.
\end{abstract}

Key words: radio continuum: blazar

PACS: $95.75 . \mathrm{Kk}, 98.54 . \mathrm{Cm}$

\section{Introduction}

The blazar $1156+295(z=0.729$, Véron-Cetty \& Véron (1998) $)$ is among the most active of highly polarized (HPQ) and optically violent variable (OVV) sources (Glassgold et al., 1983; Wills et al., 1983, 1992) In its active phase, this source has shown flux-density fluctuations at optical wavelengths with an amplitude of $\sim 5-7 \%$ on time scales of 0.5 hour. Also, both the position angle and fraction of optical polarization vary dramatically. At cm wavelengths $\sim 10 \%$ variations in total flux density have been observed on time scales less than several days. Finally, $1156+295$ has flared in $100 \mathrm{MeV} \gamma$-rays at least three times since 1992, while the quiescent $\gamma$-ray emission remains undetected (Mukherjee et al., 1997).

High-resolution radio imaging has revealed arcsecond and milliarcsecond structure consistent with the activity described above as well as with the standard blazar model of a jet aligned nearly along the line of sight. 
On the arcsecond scale, the $1.5 \mathrm{GHz}$ VLA image shows a symmetrical structure in the north-south direction (Antonucci \& Ulvestad, 1985). The MERLIN image at $1.6 \mathrm{GHz}$ and the VLA image at $5 \mathrm{GHz}$ (McHardy et al., 1990) show extended emission on the arcsecond-scale and a knotty jet of length $\sim 2$. 5 at p.a. $-19^{\circ}$. The northern end of the jet has a hotspot, at which point it turns through about $90^{\circ}$ to the east, ending in a diffuse region of size $\sim 11^{\prime \prime} 5$. There is a region of similar diffuse emission to the south about $2^{\prime \prime}$ from the core, at p.a. $\sim 200^{\circ}$. From the maps, $1156+295$ appears to resemble a classical double radio source seen end-on, with its northern jet relativistically beamed towards us, and hence dominating over any southern jet, while the diffuse emission is not beamed and appears symmetric.

Based on VLBI observations at three frequencies, McHardy et al. (1990, 1993) estimated the apparent superluminal velocity of $26 h^{-1} c$. This is much larger than any proper motion reliably found for any other source $-\sim 2.5$ times greater than that of the next fastest object in its redshift bin on the $\mu-z$ diagram (Vermeulen \& Cohen, 1994). Such a high apparent proper motion in principle could be a projection effect: when the jet axis is aligned near the line of sight, small changes in the direction of the jet axis can produce large changes in the apparent proper motion. Lower apparent velocities in the range of 3.5$8.8 h^{-1} c$ were reported on the basis of ten epochs of geodetic observations at 8.4 and $2.3 \mathrm{GHz}$ (Piner \& Kingham, 1997). Arguments against highly beamed synchrotron emission from the milliarcsecond core (thus, not necessarily a small angle between the very inner part of the jet and viewing directions) have been reported on the basis of the VSOP Space VLBI observations at 1.6 $\mathrm{GHz}$ with the angular resolution of $4.4 \times 1.4$ mas (Hirabayashi et al., 1998).

In this paper, we discuss the observations of the blazar $1156+295$ at $5 \mathrm{GHz}$ with the EVN + MERLIN (February 1997) in comparison with the earlier VLBA observation. Throughout this paper, the values $H_{0}=100 h \mathrm{~km} \mathrm{~s}^{-1} \mathrm{Mpc}^{-1}$, $q_{0}=0.5$, and $S \propto \nu^{\alpha}$ will be used.

\section{The observations and data reduction}

A full-track 12-hour EVN + MERLIN observation of the blazar 1156+295 was carried out at $5 \mathrm{GHz}$ in February 1997. The MERLIN array comprised 6 antennas (Defford, Cambridge, Knockin, Darnhall, Mark 2, and Tabley). The central wavelength was $4994 \mathrm{MHz}$ and the observing bandwidth was $14 \mathrm{MHz}$. The amplitude calibration was carried out at Jodrell Bank with the calibrator source OQ208. The imaging was done using the NRAO Astronomical Image Processing System (AIPS) package.

The EVN array comprised Effelsberg, WSRT, Jodrell Bank (Mark 2), Cam- 
bridge, Onsala, Medicina, Torun, Shanghai, Urumqi, and Hartebeesthoek. The data were acquired with the Mk III VLBI recording system in Mode B with an effective bandwidth of $28 \mathrm{MHz}$ and correlated at the Max-Plank Institut für Radioastronomie in Bonn. No fringes were found on the baselines to Urumqi. The EVN data were calibrated and fringe-fitted using the NRAO AIPS package. The initial amplitude calibration was accomplished using the system temperature measurements made during the observations and the a priori station gain curves.

The snapshot-mode observation of $1156+295$ with the VLBA at $5 \mathrm{GHz}$ was made in June 1996 as part of the VSOP prelaunch VLBA Survey (Edwards \& Fomalont, 1998). Eight intermediate frequency channels, each $8 \mathrm{MHz}$ wide, were recorded for a total bandwidth of $64 \mathrm{MHz}$. The data were correlated at the NRAO VLBA correlator (Socorro, NM, USA). The fringe-fitted and calibrated VLBA data were kindly made available to us by the VSOP/VLBA pre-launch survey group (Edwards \& Fomalont, 1998).

The post-processing — including editing, phase and amplitude self-calibration, imaging and model fitting — was performed within AIPS for both the EVN and VLBA data sets.

\section{Results}

Our MERLIN image shows traces of a straight jet at the position angle of $-20^{\circ}$ and $2^{\prime \prime}$ in length (Hong et al., in preparation). This is consistent with the lower-resolution VLA and MERLIN images reported by McHardy et al. (1990). There appear to be several regularly spaced knots within 1 arcsecond of the core, possibly indicating quasi-periodic activity that has propagated from the base of the jet.

Because of missing Urumqi data, the $\mathrm{u}-\mathrm{v}$ coverage of the EVN data has a big gap between 25 and $90 \mathrm{M} \lambda$. Therefore, we imaged with the data of the European telescopes first. The image (Fig. 1) reveals a jet that is bending from North to North-East before turning back to the North, presumably to meet the arcsecond scale jet.

The image of VLBA presents a similar structural pattern (Fig. 1). It shows more clearly that the jet is bent from the North to East and then back to the North-East.

A higher resolution EVN image was obtained by using all the data, including data from Hartebeesthoek and Shanghai (Hong et al., in preparation). The results show that a new jet component starts at about p.a. $50^{\circ}$ before bending 

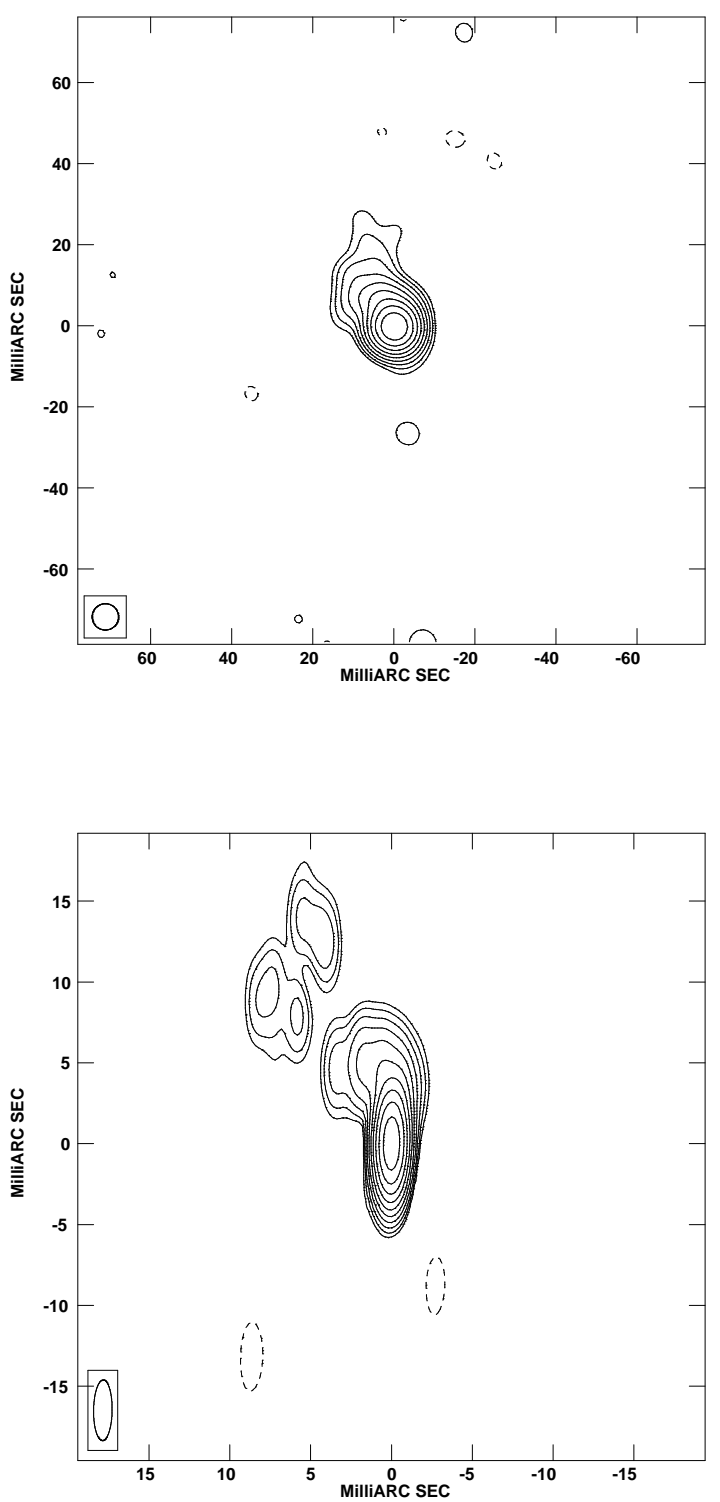

Fig. 1. The EVN (top) and VLBA (bottom) images of $1156+295$ at 5 GHz. Note the different scales of the two maps. Contour levels are $8.0 \times 10^{-4}(-3,3,6,12,24$, 48, 96, 192, 384, 768, 1536). The peak flux densities are 1.18 and $2.08 \mathrm{Jy} / \mathrm{beam}$, respectively.

to the North within 2 milliarcseconds of the core, which is consistent with the $8 \mathrm{GHz}$ images (Piner \& Kingham, 1997). In Piner and Kingham's images, one can also note that the jet components start at the position angle of $45^{\circ}$, then move clockwise to the North before bending to the East sharply. 


\section{Discussion}

We note that the peak flux density at two epochs varies from $2.09 \mathrm{Jy} / \mathrm{beam}$ for the VLBA map (epoch 1996.43) to $1.2 \mathrm{Jy} /$ beam for the later EVN map (epoch 1997.14), which is clear evidence of the decrease of the core brightness (the VLBA synthesized beam of Fig. 1 is smaller than that of EVN, Fig. 1). From the data of the University of Michigan Radio Astronomy Observatory, the total flux densities at 14.5, 8.0, and $4.8 \mathrm{GHz}$ have all decreased from mid of 1996 to early 1997 (UMRAO database, 1998). In $15 \mathrm{GHz}$ VLBA maps, the peak flux density decreased from $1.95 \mathrm{Jy} /$ beam (16 May 1996) to $0.67 \mathrm{Jy} / \mathrm{beam}$ (13 March 1997) (Kellermann et al., 1998). All these indicate clearly that the flare of $1156+295$ was due to the outburst of the central core component. At that time a new jet component was ejected.

Helical jets have been proposed to explain the bi-modal distribution of the difference between arcsecond and milliarcsecond structural axes observed for core-dominated radio sources (Conway \& Murphy, 1993). The helical pattern could result from the precession of the base of the jet (e.g. Linfield (1981)) or fluid-dynamical instabilities in the interaction between the jet material and surrounding medium (Hardee, 1987). The structural oscillations at mas scales can be explained by the orbital motion of a binary black hole (e.g. Roos et al. (1993) for the case of $1928+738)$.

The jet of $1156+295$ has a structural oscillation on the mas scale and a large $\Delta$ p.a. between its pc-scale and the kpc-scale directions, which may indicate that the oscillation of the jet is a projection effect of a helical jet. The observed properties of the jet could be explained by two effects: the precession of the spin axis of the black hole emitting the jet and the orbital motion of a binary black hole. In this case, the jet of $1156+295$ could be ejected in the direction very closely aligned to the line of sight. The jet then would have a high Doppler boosting but would demonstrate a relatively low apparent proper motion. As the direction of jet curves away from the line of sight, the Doppler factor will decrease (thus, flux density will decrease also), but the apparent proper motion velocity will increase. As the bright radio component moves outward, it will reach the viewing angle of the maximum apparent transverse velocity, while its Doppler boosting factor will continue to monotonically decrease. This qualitative model can explain the evolution of the outburst in the core (McHardy et al., 1990) and the apparent initial acceleration of the proper motion of the jet components (Piner \& Kingham, 1997). It is also generally consistent with the $\gamma$-ray observation at the $\mathrm{GeV}$ band: the source demonstrated several short active periods at the high-energy $\gamma$-ray band but remained in a quiescent state most of the time between flares (Mukherjee et al., 1997). The model suggests that the $\gamma$-ray flares may preceed radio outbursts since the former require higher Doppler boosting. 
Acknowledgements. This research was supported by the National Science Foundation and the Pan Deng Plan of China. XYH thanks JIVE for the hospitality during his visit in 1998. LIG acknowledges partial support from the European Comission TMR programme, Access to Large-Scale Facilities under contract ERBFMGECT950012. The authors are grateful to the staff of EVN, MERLIN and VLBA for support of the observing projects. The authors express their gratitude to the team of the VSOP/VLBA pre-launch survey, particularly Ed Fomalont and Phil Edwards, for the permission to use their data. This research has made use of data from the University of Michigan Radio Astronomy Observatory, which is supported by the National Science Foundation and by funds from the University of Michigan. The National Radio Astronomy Observatory is operated by Associated Universities, Inc. under a Cooperative Agreement with the National Science Foundation.

\section{References}

Antonucci, R.R. \& Ulvestad, J.S., 1985, ApJ, 294, 158.

Conway, J. \& Murphy D. W., 1993, ApJ, 411, 89.

Edwards, P.G. \& Fomalont, E.B., 1998, in Radio Emission from Galactic and Extragalactic compact sources, eds. J.A.Zensus, G.B.Taylor, J.M.Wrobel, ASP Conf. Series v. 144, 143.

Glassgold, A.E., Bergman, J.M., Huggins, P.J., Kinney, A.L., Pica, A.J., \& Pollock, T.J., et al. 1983, ApJ, 274, 101.

Hardee, P. E., 1987, ApJ, 318, 78.

Hirabayashi, H. et al., 1998, Science, 281, 1825.

Kellermann, K.I., Vermenlen, R.C., Zensus, J.A., \& Cohen, M.H., 1998, AJ, $115,1295$.

McHardy, I.M., Marscher, A.P., Gear, W.K., Muxlow, T., Lehto, H.J., \& Araham, R.G., 1990, MNRAS, 246, 305.

McHardy, I.M., Marscher, A.P., Gear, W.K., Muxlow, T., Lehto, H.J., \& Araham, R.G., 1993, MNRAS, 261, 464.

Mukherjee, R., Bertsch, D.L., Bloom, S.D., Dingus, B.L., Esposito, J.A., \& Fitchel, R.C., et al., 1997, ApJ, 490, 116.

Linfield, R. P., 1981, ApJ, 250, 464.

Piner, B. G. \& Kingham, K., 1997, ApJ, 485, L61.

Roos, N., Kaastra, J. S. \& Hummel C. A., 1993, ApJ, 409, 130.

University of Michigan Radio Astronomy Observatory database, http://www .astro.lsa.umich.edu/obs/radiotel/umrao.html.

Vermeulen, R. C. \& Cohen, M. H., 1994, ApJ, 430, 467.

Véron-Cetty, M.-P. \& Véron, P., 1998, A Catalogue of Quasars and Active Galactic Nuclei (8th edition), ESO Sci. Report No. 18.

Wills, B.J., Wills, D., Breger, M., Antonucci, R.R., \& Barvainis, R.E., 1983, ApJ,274, 62.

Wills, B.J., Pollock, J.T., Aller, H.D., Aller, M.F., Balonek, T.J., \& Barvainis, R.E., et al., 1992, ApJ, 398, 454. 